\title{
Osseous choristoma of the tongue: A case report with dermoscopic study
}

\author{
HITOSHI YOSHIMURA $^{1}$, SEIGO OHBA ${ }^{1,2}$, YOSHIAKI IMAMURA ${ }^{3}$ and KAZUO SANO ${ }^{1}$ \\ ${ }^{1}$ Department of Dentistry and Oral Surgery, Unit of Sensory and Locomotor Medicine, \\ Division of Medicine, Faculty of Medical Sciences, University of Fukui, Fukui 910-1193; \\ ${ }^{2}$ Department of Regenerative Oral Surgery, Graduate School of Biomedical Sciences, Nagasaki University, \\ Nagasaki 852-8521; ${ }^{3}$ Division of Surgical Pathology, University of Fukui Hospital, Fukui 910-1193, Japan
}

Received September 20, 2017; Accepted November 27, 2017

DOI: $10.3892 / \mathrm{mco} .2017 .1523$

\begin{abstract}
Osseous choristomas are rare benign lesions characterized by ectopic bone formation in the soft tissue of the head and neck region. Dermoscopy visualizes the morphological characteristics that are not observable by the naked eye, and may be used for the evaluation of calcification under the skin. The present study reports a case of an osseous choristoma arising in the tongue, and reveals the dermoscopic features of osseous choristoma from a surgical specimen. A 7-year-old boy was referred to the Department of Dentistry and Oral Surgery, with an asymptomatic pedunculated mass in the tongue. The lesion was removed completely with the adjacent normal tissue under general anesthesia. Dermoscopy of the surgical specimen revealed the hypovascular and homogeneous pattern of the lesion with round extruded whitish material. Based on dermoscopic findings, the presence of calcified hard tissue in the submucosa was verified by the dermatologist. Radiographic examination of the surgical specimen revealed the lesion contained a radiopaque trabeculated mass. Histologically, the lesion contained an osseous tissue, and the pathological diagnosis of osseous choristoma was made following consideration of the ectopic bony tissues that were localized away from the maxillo-mandibular bone. The postoperative course was uneventful with no signs of recurrence during the 36 months following surgery. To the best of the author's knowledge, this is the first report of evaluation of osseous choristoma using dermoscopy. The observation indicates the usefulness of dermoscopy for the diagnosis of oral ossified lesion in oral soft tissue.
\end{abstract}

Correspondence to: Dr Hitoshi Yoshimura, Department of Dentistry and Oral Surgery, Unit of Sensory and Locomotor Medicine, Division of Medicine, Faculty of Medical Sciences, University of Fukui, 23-3 Matsuokashimoaizuki, Eiheiji, Yoshida, Fukui 910-1193, Japan

E-mail: omfs@u-fukui.ac.jp

Key words: osseous choristoma, tongue, ectopic bone formation, soft tissue, ossification, dermoscopy

\section{Introduction}

Osseous choristomas are rare benign lesions characterized by ectopic bone formation in the soft tissue of the head and neck region $(1,2)$. They occur more frequently in the lingual region, and there is a variety of the differential diagnosis including the lesion of hard tissue in soft tissue (3-7). Dermoscopy is a non-invasive diagnostic method that allows an in vivo assessment of morphologic features, which are not visible to the naked eye (8). This method can be regarded as a link between clinical and histopathologic examination, and is increasingly used in general dermatology (8). Vascular structures, color variegation, follicular abnormalities, and specific features are the main criteria to be considered (9). Dermoscopy is a valuable tool that improves diagnostic accuracy (9). It is commonly used for the evaluation of pigmented skin lesions $(10,11)$, and also could be used for the evaluation of calcification under the skin (12-14). In this paper, we report a case of osseous choristoma arising in the tongue. We also examine the dermoscopic features of osseous choristoma from surgical specimen and evaluate its usefulness for the diagnosis of osseous choristoma. Furthermore, we review the relevant literature and discuss the pathophysiological mechanism responsible for ossification in soft tissue.

\section{Case report}

A 7-year-old Japanese boy was referred in August 2012 to the Department of Dentistry and Oral Surgery, University of Fukui Hospital for an evaluation of a mass in the tongue. The patient's medical history revealed the presence of autism. Physical examination revealed a non-tender pedunculated mass covered with normal mucosa in the posterior portion of the tongue (Fig. 1). The lesion was approximately $5 \mathrm{~mm}$ in diameter. The mass was movable, and there was no evidence of adhesion to the surrounding tissues. The patient had no history of inflammation or trauma in the region. Sensory disturbance was not evident. Magnetic resonance imaging (MRI) revealed a $5 \mathrm{~mm}$, well-circumscribed mass in the tongue region (Fig. 2). The mass exhibited homogeneous low signal intensity on T1and T2-weighted images. MRI also exhibited a normal thyroid gland in shape and position. The clinical diagnosis was a 
benign tumor in the tongue. The patient did not complain of any symptoms and was placed under observation. The lesion was slightly enlarged during 2 years of follow-up. Then, in August 2014, the lesion was removed completely with the adjacent normal tissue under general anesthesia. The surgical specimen was sized at $6 \mathrm{~mm}$ (Fig. 3A). Dermoscopy of the surgical specimen revealed a hypovascular and homogeneous pattern of the lesion with round extruded whitish material (Fig. 3B). Based on dermoscopic findings, the presence of calcified hard tissue in submucosa was revealed by our dermatologist. Radiographic examination of the surgical specimen showed the lesion containing a radiopaque trabeculated mass (Fig. 3C). Histological examination revealed that the mass consisted of well-circumscribed bone, and it was surrounded by stratified squamous epithelium (Fig. 4A). The bone tissue had lamellar structures. Osteoblasts and osteocytes were found on the bone surface and in the bone lacunae, but osteoclasts were not observed (Fig. 4B). There was no evidence of inflammation or malignancy in the specimen. No thyroid tissue was found. The histological diagnosis of an osseous choristoma was made after consideration of the ectopic bony tissues that were localized away from the maxillo-mandibular bone (3). The postoperative course was uneventful. No signs of recurrence were found during the 36 months of the follow-up examination. The patient provided informed consent for the use of the data in this study.

\section{Discussion}

The term of 'choristoma' defines a tumor-like lesion that is composed of normal tissue in an abnormal region $(1,2)$. Choristomas are termed according to the tissues from which they are derived; they have occurred from osseous, cartilaginous, lingual thyroid, salivary gland, glial, gastric mucosal tissues and other tissue (2). The term 'osseous choristoma' was used by Krolls et al to define soft tissue osteomas in the head and neck region (3).

To date, ninety-seven cases of osseous choristomas of the oral and maxillofacial region have been reported in the English-language literature. In our literature review, the mean patient age was 32 years, ranging from 5 to 86 years. More than $70 \%$ of these lesions have been reported in women. The size of the lesions ranged from 5 to $50 \mathrm{~mm}$ in diameter. The location and frequency of the lesions were as follows; tongue (76 cases: 78\%), buccal mucosa (14 cases: 15\%), alveolar mucosa ( 2 cases: $2 \%$ ), submandibular region ( 2 cases: $2 \%$ ), submental region (1 case: $1 \%$ ), masseter muscle (1 case: $1 \%$ ), and hard plate ( 1 case: $1 \%$ ). In the case of the tongue, the most frequent affected region is the posterior third of the tongue dorsum near the foramen caecum and circumvallate papillae. A symptoms of a lump, dysphagia, pain, gagging and nausea have been reported.

In clinical findings, osseous choristomas are observed as either a sessile or a pedunculated mass (2). The clinical differential diagnosis includes other tumor-like lesions, such as salivary gland tumors, fibromas, lipomas, neural tumors, giant cell tumors, and soft tissue cysts $(4,5)$. If soft tissue calcification and bone formation are observed, the differential diagnosis should include teratomas, osteomas, calcified lymph nodes, osseous hamartoma, calcified hamartomas

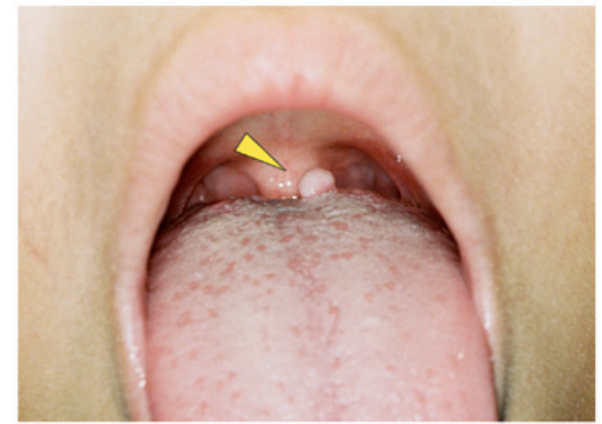

Figure 1. Clinical appearance. Intraoral view showing a swelling of the left lingual mucosa (arrowhead). The pedunculated mass was covered with normal mucosa.

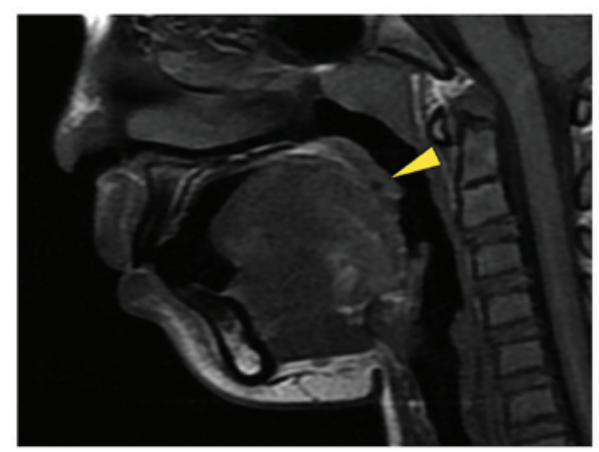

Figure 2. MRI finding. Sagittal MR image showing a well-circumscribed lobular mass of $5 \mathrm{~mm}$ in diameter in the tongue base (arrowhead).

and osteolipomas $(6,7)$. When the lesion is located close to the foramen caecum, the lingual thyroid nodule should be considered. Osseous choristomas may also occur in many pathological conditions, such as hyperparathyroidism and skin inflammation (3).

The dermoscopy significantly improves the diagnostic accuracy of skin lesions (14), and in consequence, dermoscopy is now an integral part of the clinical skin examination (13). Compared to radiological and histological examinations, dermoscopy can detect even small lesions without invasion or side effects, and it might contribute to earlier treatment. This method is also effective for evaluating the borders of pathologic lesions. This in turn might improve the treatment outcomes. Dermoscopy could also be used for the evaluation of calcification under the skin (12-14). Recently, the usefulness of this technique for the evaluation of oral soft tissue lesions, including those on the tongue, has been reported (15-18). To identify the characteristics of the lesion, we performed a dermoscopic study. In our case, dermoscopic examination could not be performed before resection because the instrument could not reach the lesion directly. The resected specimen was evaluated, and a hypovascular and homogeneous pattern of the lesion with round extruded whitish material was observed. The feature was consistent with the previously described dermoscopic pattern for calcification of skin lesion (12-14). Based on dermoscopic findings, the presence of calcified hard tissue in the submucosa was suggested by our dermatologist. Actually, the calcified hard tissue was confirmed by radiographic finding, and whitish structure in 

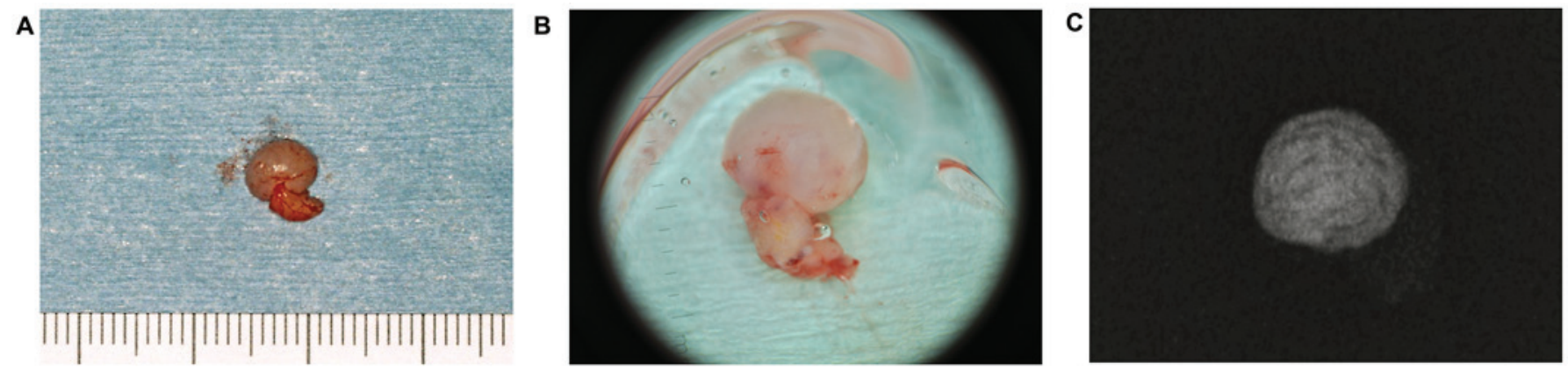

Figure 3. Dermoscopic and radiological findings of the surgical specimen. (A) The surgical specimen was sized at 6 mm. (B) Dermoscopy of the surgical specimen showing a hypovascular and homogeneous pattern of the lesion with round extruded whitish material. (C) Radiograph of the surgical specimen showing a radiopaque trabeculated mass in the lesion.
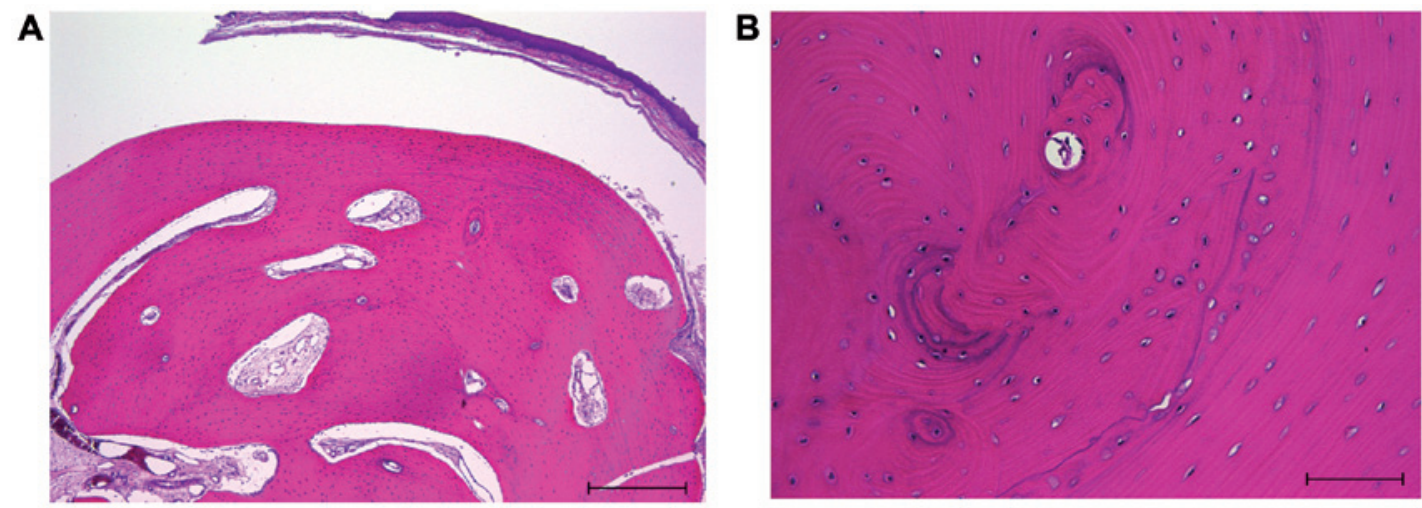

Figure 4. Microphotograph of the surgical specimen. (A) The mass consisted of well-circumscribed bone and was surrounded by stratified squamous epithelium (hematoxylin and eosin, scale bar $500 \mu \mathrm{m}$ ). (B) Osteoblasts were found on the bone surface, and osteocytes were found in the bone lacunae (hematoxylin and eosin, scale bar $100 \mu \mathrm{m})$.

dermoscopic finding was histopatologically corresponded to osseous choristoma. As far as we know, this is the first report of dermoscopic evaluation of oral calcified lesion in the soft tissue, and the usefulness of dermoscopy could be confirmed. If lesions existed in the anterior oral portion, dermoscopy might be beneficial in establishing preoperative diagnosis of osseous choristoma. We have shown the dermoscopic features of osseous choristoma, but this is a case report and dermoscopic criteria of osseous choristoma has not been established yet. Intraoral dermoscopy is still less investigated and less popular among clinicians. There is a need to intensify examination, which would result in creating clear-cut dermoscopic criteria including other than this lesions. Dermoscopy might be utilized as an adjunctive device, but a limiting factor is the accessibility to the lesion. Previous reports of dermoscopical evaluation were mostly performed in the lesion of tongue or lip. The future technology would focus on developing a miniaturized, flexible dermoscope that will allow detailed examination of the whole oral cavity.

MRI is also one of the useful imaging modalities in the head and neck region. To the best of our knowledge, only four cases of osseous choristomas that were evaluated with MRI have been reported $(6,19-21)$. Lee et al (19) indicated that low signal intensity on both T1- and T2-weighted images without enhancement with contrast medium reflects the ossific and calcific nature of the lesion. If the existence of hard tissue is suspected, X-ray examination and computed tomography
(CT) are also useful, as their results can yield further diagnostic confidence (19). In our case, CT examination was not performed in consideration of the effect of radiation exposure. However, X-ray examination of the surgical specimen could show the radiopaque trabeculated mass in the lesion.

Histologically, the lesions are generally constituted by a well-circumscribed, lamellated mass of dense, vital bone with Haversian canals, circumscribed the fibrous connective tissue (2). Osteocytes are observed in the lacunae within the bony spherules (2). Occasionaly, the mass may contain blood vessels lined with fibrous tissue or endothelium and several cells that are similar to the osteoclasts (3). In our case, the lesion contained well-circumscribed bone, and it was surrounded by stratified squamous epithelium. Osteoblasts and osteocytes were observed on the outer surface of bony trabeculae and in the lacunae of the formed bone, respectively. Nevertheless, osteoclasts were not found in the formed bone. In our case, a slight increase of the size was observed during 2 years of follow-up. These results indicate that bone formation slowly occurred in the lesion.

The pathogenesis of osseous choristomas remains controversial. Various reports have suggested that the lesions originate via the ossification of the branchial arch remnants (22), epignathus formation and degenerating ossifying fibroma (23), and metabolic alterations in pluripotent mesenchymal cells as a response to an unknown stimulus (24). In our previous report, immunohistochemical examination of BMP-2 and -4 was 
performed to investigate the molecular mediators of ectopic bone formation and presented the evidence of the expression of BMP-2 and -4 in an osseous choristoma (20). BMPs belong to the transforming growth factor (TGF) $-\beta$ superfamily and are essentially involved in embryogenesis, skeletal formation, hematopoiesis and neurogenesis (25). These proteins promote differentiation of mesenchymal cells into osteoblasts and chondorocytes and play critical roles in bone development and metabolism (25). Notably, BMP-2 and -4 have been reported to play an important role in ectopic bone formation in an experimental animal model and clinical research (25-28). Our previous report indicates that the expression of BMP-2 and -4 is associated with the ossification of osseous choristomas. Thus, there is a possible mechanism of ectopic bone formation; BMPs secreted from the lesion may trigger ectopic ossification in the soft tissue by stimulating pluripotent mesenchymal progenitor cells to differentiate into bone-forming cells.

Osseous choristomas are best treated by local surgical excision (1). In our case, no evidence of recurrence has appeared at 36 months postoperatively.

\section{Acknowledgements}

The authors wish to thank Dr. Wataru Takashima, Department of Dermatology, Unit of Sensory and Locomotor Medicine, Division of Medicine, Faculty of Medical Sciences, University of Fukui, for his support in dermoscopic diagnosis, and Dr. Minako Shimada, Department of Dentistry and Oral Surgery, Unit of Sensory and Locomotor Medicine, Division of Medicine, Faculty of Medical Sciences, University of Fukui, Fukui, for her help in the treatment.

\section{References}

1. Neville BW, Damm DD, Allen CM and Bouquot JE (eds): Oral and Maxillofacial Pathology. 3rd edition. Saunders Elsevier, St. Louis, 552, 2009.

2. Chou LS, Hansen LS and Daniels TE: Choristomas of the oral cavity: A review. Oral Surg Oral Med Oral Pathol 72: 584-593, 1991.

3. Krolls SO, Jacoway JR and Alexander WN: Osseous choristomas (osteomas) of intraoral soft tissues. Oral Surg Oral Med Oral Pathol 32: 588-595, 1971.

4. Tohill MJ, Green JG and Cohen DM: Intraoral osseous and cartilaginous choristomas: Report of three cases and review of the literature. Oral Surg Oral Med Oral Pathol 63: 506-510, 1987.

5. Psimopoulou $\mathrm{M}$ and Antoniades K: Submental osseous choristoma: A case report. J Oral Maxillofac Surg 56: 666-667, 1998.

6. Dalkiz M, Hakan Yurdakul R, Pakdemirli E and Beydemir B: Recurrent osseous choristoma of the masseter muscle: Case report. J Oral Maxillofac Surg 59: 836-839, 2001.

7. Yamamoto N, Ishikawa A, Yamauchi K, Miyamoto I, Tanaka T, Kito S, Matsuo K, Yamashita Y, Morimoto Y and Takahashi T: Osteolipoma of the lower lip: A case report. Asian J Oral Maxillofac Surg 23: 143-145, 2011.

8. Russo T, Piccolo V, Lallas A and Argenziano G: Recent advances in dermoscopy. F1000Res 5: 184, 2016.

9. Lallas A, Zalaudek I, Argenziano G, Longo C, Moscarella E, Di Lernia V, Al Jalbout S and Apalla Z: Dermoscopy in general dermatology. Dermatol Clin 31: 679-694, 2013.
10. De Giorgi V, Massi D and Carli P: Dermoscopy in the management of pigmented lesions of the oral mucosa. Oral Oncol 39: 534-535, 2003.

11. Olszewska M, Banka A, Gorska R and Warszawik O: Dermoscopy of pigmented oral lesions. J Dermatol Case Rep 2: 43-48, 2008

12. Strumia R: Videodermatoscopy: A useful tool for diagnosing cutaneous dystrophic calcifications. Dermatol Online J 11: 28 , 2005.

13. Lallas A, Moscarella E, Argenziano G, Longo C, Apalla Z, Ferrara G, Piana S, Rosato S and Zalaudek I: Dermoscopy of uncommon skin tumours. Australas J Dermatol 55: 53-62, 2014.

14. Zaballos P, Llambrich A, Puig S and Malvehy J: Dermoscopic findings of pilomatricomas. Dermatology 217: 225-230, 2008.

15. Okamoto T, Sasaki R, Kataoka T, Kumasaka A, Kaibuchi N Naganawa T, Fukada K and Ando T: Dermoscopy imaging findings in the normal Oral Mucosa. Oral Oncol 51: e69-e70, 2015.

16. Warszawik-Hendzel O, Słowińska M, Olszewska M and Rudnicka L: Melanoma of the oral cavity: Pathogenesis, dermoscopy, clinical features, staging and management. J Dermatol Case Rep 8: 60-66, 2014.

17. Güleç AT: Dermoscopic features of squamous cell carcinoma of the tongue: It looks similar to cutaneous squamous cell carcinoma. J Am Acad Dermatol 75: e53-e54, 2016.

18. Drogoszewska B, Chomik P, Polcyn A and Michcik A: Clinical diagnosis of oral erosive lichen planus by direct oral microscopy. Postepy Dermatol Alergol 31: 222-228, 2014.

19. Lee DL, Wong KT, Mak SM, Soo G and Tong MC: Lingual osteoma: Case report and literature review. Arch Otolaryngol Head Neck Surg 135: 308-310, 2009.

20. Yoshimura H, Ohba S, Matsuda S, Kobayashi J, Ishimaru K, Imamura Y and Sano K: Osseous choristoma of the buccal mucosa: A case report with immunohistochemical study of bone morphogenetic protein-2 and -4 and a review of the literature. J Oral Maxillofac Surg Med Pathol 26: 351-355, 2014.

21. Yamamoto M, Migita M, Ogane S, Narita M, Yamamoto N, Takaki T, Matsuzaka K and Shibahara T: Osseous choristoma in child with strong vomiting reflex. Bull Tokyo Dent Coll 55: 207-215, 2014.

22. Monserrat M: Ostéome de la langue. Bull Soc Anat 88: 282-283, 1913.

23. Church LE: Osteoma of the tongue. Report of a case. Oral Surg Oral Med Oral Pathol 17: 768-770, 1964.

24. Roy JJ, Klein HZ and Tipton DL: Osteochondroma of the tongue. Arch Pathol 89: 565-568, 1970.

25. Xiao YT, Xiang LX and Shao JZ: Bone morphogenetic protein. Biochem Biophys Res Commun 362: 550-553, 2007.

26. Bragdon B, Moseychuk O, Saldanha S, King D, Julian J and Nohe A: Bone morphogenetic proteins: A critical review. Cell Signal 23: 609-620, 2011.

27. Kusumoto K, Bessho K, Fujimura K, Akioka J, Ogawa Y and Iizuka T: Comparison of ectopic osteoinduction in vivo by recombinant human BMP-2 and recombinant Xenopus BMP-4/7 heterodimer. Biochem Biophys Res Commun 239: 575-579, 1997.

28. Kim SY, Choi HY, Myung KB and Choi YW: The expression of molecular mediators in the idiopathic cutaneous calcification and ossification. J Cutan Pathol 35: 826-831, 2008.

This work is licensed under a Creative Commons Attribution-NonCommercial-NoDerivatives 4.0 International (CC BY-NC-ND 4.0) License. 